\title{
Influencia del método de limpieza empleado previo el empleo de materiales ionomericos en microfiltración de cavidades a nivel cervical
}

\author{
Influence of the cleaning method employed prior to the use of ionomeric materials in micro \\ filtration of cavities at the cervical level
}

María Muñoz Estrella ${ }^{1 a}$, Alexander Cruz Gallegos ${ }^{2 a}$, Ana del Carmen Armas Vega ${ }^{1 a, b}$

\section{RESUMEN}

Objetivo: Evaluar la influencia que produce la limpieza de la cavidad con óxido de aluminio y piedra pómez sobre la microfiltración marginal cuando se emplean materiales lonoméricos (IV) Materiales y Métodos: En 40 dientes premolares humanos, fueron preparadas cavidades a nivel del límite amelo cementario, los dientes fueron divididos aleatoriamente en 4 grupos $(\mathrm{n}: 10)$ recibieron procedimientos de limpieza y restauración según el grupo, G1 limpieza con aire abrasivo y restauración con IV Ketac Molar Easy Mix (IVKM), G2 aire abrasivo y restauración con IV Gold Label 2 (IVGL), G3 piedra pómez e IVKM, G4 piedra pómez e IVGL. Tras proceso de envejecimiento térmico, pigmentación con azul de metileno y corte en sentido buco lingual, los fragmentos con mayor integridad fue analizado al estereomicroscopio por tres evaluadores quienes establecieron el grado de micro filtración a nivel cervical y oclusal; la media obtenida por superficie por diente fue analizada estadisticamente. Resultados: el análisis ejecutado revelo normalidad en los datos, encontrando mediante el test de Tukey que todos los grupos son iguales, sin diferencia en cuanto al tratamiento y al material utilizado. Conclusiones: se evidencio micro filtración en todos los grupos, independientemente del tratamiento de limpieza y el material de restauración empleado, de forma más notoria a nivel de la pared cervical.

Palabras Clave: Cementos de ionómero Vitreo; Filtración Dental; Óxido de Aluminio. (Fuente: DeCS BIREME)

\section{ABSTRACT}

Objective: To evaluate the influence of the cleaning of the cavity with aluminum oxide and pumice on the marginal microfiltration when using ionomeric materials (IV). Materials and Methods: In 40 human premolar teeth, cavities were prepared at the level of the cemetery amelo, the teeth were randomly divided into 4 groups ( $\mathrm{n}: 10)$ received cleaning and restoration procedures according to the group, G1 cleaning with abrasive air and restoration with IV Ketac Molar Easy Mix (IVKM), G2 abrasive air and restoration with IV Gold Label 2 (IVGL), G3 pumice stone and IVKM, G4 pumice stone and IVGL. After the process of thermal aging, pigmentation with methylene blue and cutting in the lingual buccal direction, the fragments with the highest integrity were analyzed to the stereomicroscope by three evaluators who established the degree of micro filtration at the cervical and occlusal levels; the average obtained by surface per tooth was analyzed statistically. Results: The analysis performed revealed normality in the data, finding through the Tukey test that all groups are equal, with no difference in treatment and material used. Conclusions: Micro filtration was evident in all the groups independent of the cleaning treatment and the restoration material used, most notably at the level of the cervical wall.

Key words: Vitreo ionomer cements; Dental Filtration; Aluminum Oxide. (Source: MeSH NLM)

Recibido: 20 de febrero de 2019

${ }^{1}$ Universidad Central del Ecuador.

2 Universidad Tecnológica Equinoccial.

a Odontólogo.

${ }^{\mathrm{b}}$ Docente

Correspondencia:

Correo electrónico: alexandercruz1993@hotmail.com

Publicado: 06 de julio de 2019

Este es un artículo Open Access distribuido bajo la licencia Creative Commons Atribución-NoComercialCompartirlgual 4.0

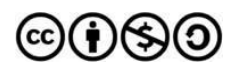




\section{INTRODUCCIÓN}

Las lesiones a nivel del tercio cervical tienen alta prevalencia relacionada a la diversidad de factores que las desencadenan, (1) los restauradores en ellas ejecutados resultan complejos considerando la relación con tejidos blandos especialmente a nivel de los márgenes cervicales, en donde la humedad puede provocar baja energía superficial dificultando una correcta adhesión ${ }^{(2)}$ y la estructura de los tejidos de la zona que dificulta conseguir una óptima adhesión entre el material y el diente, reducir el grado de microfiltración, la sensibilidad, caries recurrente y la decoloración subsecuente del material ${ }^{(3)}$.

549.45

Los cementos de ionómero de vidrio se muestran como materiales óptimos debido a su liberación de fluoruros y su capacidad de adhesión a la estructura dentaria evitando así la microfiltración ${ }^{(4)}$. La limpieza de las superficies como procedimiento previo a su colocación, busca remover restos del smear layer formados al instrumentar y mejorar por tanto la adhesión (3), muchos materiales han sido desarrollados con este fin cada uno con ventajas y desventajas (4), considerando la ausencia de un consenso en la literatura y la gran cantidad de alternativas existentes en el mercado, este estudio busca evaluar la influencia que ejerce la ejecución de limpieza de la cavidad con piedra pómez o aire abrasivo con partículas de aluminio previo a la colocación de ionomero de vidrio como material restaurador.

El ionómero de vidrio es un material recomendado al momento de restaurar lesiones iniciales de caries, por su capacidad para liberar fluoruros, adherirse químicamente a la estructura dental y potencial remineralización de los tejidos dentales. Como materiales de restauración presentan características físicas, químicas, biológicas y estéticas ${ }^{(5)}$.

Las cavidades a nivel cervical son quizás un tipo de restauración dental con mayor índice de fracaso y necesidad de re-intervenciones debido a su alto índice de fractura, exigiendo distintas formas de retención y de un material con buenas propiedades físicas y mecánicas adecuadas ${ }^{(6)}$.

La capacidad de sellado a nivel de esmalte y dentina está influenciada por su humedad y baja energía superficial, en cavidades a nivel cervical constituye un reto para el odontólogo el momento de restaurarlas, los materiales empleados generalmente para estos procedimientos dependen del medio donde son colocados y al contar con amplios túbulos dentinarios en la dentina a nivel del límite esmalte cemento, se hace difícil la colocación de materiales resinosos. En ese contexto los ionómeros de vidrio se muestran como alternativas adecuadas ${ }^{(7)}$.

La limpieza de las superficies de las paredes cavilarías han sido desarrolladas buscando disminuir la micro filtración marginal. En la práctica clínica el empleo de piedra pómez es frecuente, el uso de choro de óxido de aluminio en boca son empleados con frecuencia en procesos adhesivos sin embargo pocos estudios refieren su desempeño cuando combinamos con materiales ionoméricos ${ }^{(8)}$.

El objetivo de este estudio fue evaluar la influencia de la limpieza de la cavidad con óxido de aluminio y piedra pómez, sobre la microfiltración marginal cuando se emplean materiales lonoméricos (IV)

\section{MATERIALES Y METODOS}

Fueron considerados en el estudio una muestra de 40 dientes premolares permanentes humanos extraídos por motivos ortdónticos. Tras la extracción los dientes fueron almacenados en suero fisiológico, limpios por medio de un ultrasonido scaler, ("Woodpecker" USA), para eliminar restos de tejidos blandos y cepillo profiláctico con una pasta compuesta de piedra pómez y agua destilada $5 \mathrm{~g} / 4 \mathrm{ml}$, en pieza de mano de baja velocidad (NSK - USA). por 20 segundos y lavados con agua de la jeringa triple.

En el tercio cervical de la superficie vestibular en la unión cemento-esmalte fueron elaboradas cavidades con una fresa cilíndrica de diamante de grano grueso $\mathrm{N} 0.12$ que fue cambiada cada 5 preparaciones, colocada en pieza de mano de alta velocidad ( Coxo) bajo refrigeración por aire y agua. De $4 \mathrm{~mm}$ de ancho, $4 \mathrm{~mm}$ de largo y $2 \mathrm{~mm}$ de profundidad, estandarizando su profundidad mediante una sonda periodontal CP 12 marca (Hu-Friedy - USA). Todos los dientes fueron divididos aleatoriamente en 4 grupos ( $n$ : 10) realizando los procedimientos de limpieza y restauración según el grupo.

Grupo 1 fue ejecutado procedimientos de limpieza con oxido de aluminio tamaño de partículas de $27 \mathrm{~mm}$ con una presión de 40 psi a una distancia de $5 \mathrm{~mm}$ durante 10 segundos) mediante un Microarenador (bio- art) seguido de lavado y secado con la jeringa triple. Las paredes de la cavidad confeccionada, fueron grabadas con $0.5 \mathrm{ml}$ ácido poli acrílico al 10\% por 10 segundos, seguido de lavado y secado con torunda de papel absorbible, colocando tras dosificación y mezcla Ketack Molar (3M-ESPE - USA) manipulado según el fabricante, colocado en la cavidad cuidando de no producir burbujas. Tras el endurecimiento inicial entre 4 a 5 minutos de haber sido colocado, fue aplicado sobre el material, vaselina de forma digital. 
Grupo 2. Tras la aplicación de óxido de aluminio, como se ha descrito previamente, las paredes de la cavidad fueron grabadas con $0,5 \mathrm{ml}$ de ácidooliacrílico al $10 \%$ por 10 segundos, seguido de lavado y secado con torunda de papel absorbible, colocado tras la dosificación y mezcla Gold Label 2 (FUJI) manipulado según el fabricante colocado en la cavidad cuidando de no producir burbujas. Respetando los cuidados descritos previamente.

Grupo 3. Recibieron procedimientos de limpieza con pasta de piedra pómez y agua destilada $5 \mathrm{~g} / 4 \mathrm{ml}$, aplicado mediante un cepillo profiláctico colocado en una pieza de mano de baja velocidad (NSK) durante 2 min en cada pieza dental, seguido de lavado y secado con la jeringa tripe. Las paredes de la cavidad fueron grabadas con $0,5 \mathrm{ml}$ ácido poli acrílico al $10 \%$ por 10 segundos, seguido de lavado y secado con torunda de papel absorbible, colocando tras dosificación y mezcla Ketack molar (3M- ESPE) manipulado según el fabricante colocado en la cavidad cuidando de no producir burbujas. Respetando los cuidados descritos previamente.

Grupo 4. Pasta de piedra pómez y agua destilada $5 \mathrm{~g} / 4 \mathrm{ml}$ fueron aplicados mediante un cepillo profiláctico colocado en una pieza de mano de baja velocidad (NSK) durante 2 min en cada pieza dental, seguido de lavado y secado con la jeringa tripe, las paredes de la cavidad fueron grabadas con $0,5 \mathrm{ml}$ ácido poli acrílico al $10 \%$ por 10 segundos, seguido de lavado y secado con torunda de papel absorbible, colocando tras dosificación y mezcla Gold Label 2 (FUJI) manipulado según el fabricante colocado en la cavidad cuidando de no producir burbujas. Respetando los cuidados descritos previamente.

Tras la confección de las cavidades los dientes debidamente identificados fueron colocados en agua destilada durante 24 horas a una temperatura de (27 ${ }^{\circ} \mathrm{C}$ ambiente), concluido este periodo, la humedad de cada diente fue eliminada mediante papel absorbente y pintado con esmalte de uñas por dos ocasiones en toda su superficie excepto en el lugar de la restauración. Todos los dientes fueron sometidos a un proceso de termociclado o envejecimiento artificial que consistió en baños a $5{ }^{\circ} \mathrm{C}$ por 30 segundos a
$23 \stackrel{\circ}{\circ} \mathrm{C}$ por 10 segundos y a $55^{\circ} \mathrm{C}$ por 30 segundos, estos ciclos fueron repetidos 500 veces durante 5 horas y 50 minutos (Atoui, Chinelatti 2010). Cumplido el proceso de termociclado cada diente fue sumergido en azul de metileno al $2 \%$ por 24 horas (Bona, Pinzetta, Rosa 2007), luego de lo cual fueron lavados en agua corriente y secados por medio de papel absorbente. Realizándose un corte en dirección bucolingual en el centro de las restauraciones mediante un disco de diamante (Masterdent) adaptado a un micromotor (NSK) obteniéndose dos segmentos, seleccionando para el análisis mediante una lupa Estéreomicroscopio de aumento aquel segmento con mayor integridad del material. Tres observadores debidamente calificados entrenados y estandarizados previamente con un Kappa de Cohen de 0,7 examinaron cada fragmento seleccionado en cuanto al grado de penetración del tinte en las paredes oclusal y cervical emitiendo su criterio siguiendo la escala de valores donde:

Grado 1: únicamente se evidencia microfiltración en la mitad de la restauración.

Grado 2: existe microfiltración en toda la restauración. Grado 3: la microfiltración sobrepasa la restauración.

Con los valores emitidos por los observadores fue obtenido una media que fue recolectada en una tabla de Excel, considerando el grupo, el diente, la superficie oclusal y cervical, considerándose esta media en la evaluación estadística, mediante el programa Minitab a través de ANOVA

\section{RESULTADOS}

Los datos analizados estadísticamente revelaron provenir de una población normal con un $\mathrm{P}=0,546$, realizándose un análisis ANOVA paramétrico test de Tukey a un $95 \%$ de confianza. Los resultados obtenidos indicaron ausencia de diferencia estadística entre los grupos, tanto al analizar por material restaurador empleado, como por tratamiento de limpieza ejecutado. Sin embargo, al analizar la superficie, fue evidente una diferencia entre el nivel oclusal y cervical $(p=0,000)$, lo cual indica que existe mayor grado de microfiltración a nivel cervical.

\begin{tabular}{|l|l|l|l|l|l|}
\hline \multicolumn{2}{|l|}{ Estadísticas de muestras emparejadas } \\
\hline \multicolumn{2}{|c|}{} & Media & $N^{\circ}$ & $\begin{array}{l}\text { Desviación } \\
\text { estándar }\end{array}$ & $\begin{array}{l}\text { Media de error } \\
\text { estándar }\end{array}$ \\
\hline \multirow{2}{*}{ Par 1} & OCLUSAL & 2,00 & 40 & 0,506 & 0,080 \\
\cline { 2 - 7 } & CERVICAL & 2,45 & 40 & 0,597 & 0,094 \\
\hline & & & & & \\
\hline
\end{tabular}




\begin{tabular}{|lr|}
\hline N total & 40 \\
\hline Estadístico de contraste & 171,000 \\
\hline Error estándar & 20,153 \\
\hline $\begin{array}{l}\text { Estadístico de contraste } \\
\text { estandarizado }\end{array}$ & 4,243 \\
\hline $\begin{array}{l}\text { Significación asintótica (prueba } \\
\text { bilateral) }\end{array}$ &, 000 \\
\hline
\end{tabular}

Figura1.Estadistica de muestras emparejadas oclusal y cervical. Fuente: Gabriela M.

\section{DISCUSION}

Fue evidente la microfiltración en todos los grupos estudiados, con la única diferencia entre las superficies analizadas, coincidiendo con otros estudios donde fue aplicado aire abrasivo a nivel de dentina como procedimiento de limpieza por 5 segundos ${ }^{(9)}$ asociando una obstrucción de los túbulos dentinarios por la presencia de una capa de polvo residual que interferiría en la unión del material a la estructura dental, provocando microfiltración.

Comparado el empleo de óxido de aluminio de 27 o 50 micras en cuanto a reducir la microfiltración de restauraciones resinosas, la recomendación de los autores constituye su combinación con procedimientos de grabado ácido ${ }^{(10)}$ razón por la cual fue realizado la aplicación de ácido poli acrílico como agente acondicionador en ambos materiales empleados. Si bien en nuestro estudio la aplicación del óxido de aluminio fue por un tiempo de 10 segundos y con partículas de 27 micras estas permanecieron impregnadas en la superficie y obstruyeron la adhesión del material, sobre todo en la pared cervical.

En el caso de la piedra pómez, su acción sobre esmalte desencadena un aumento de energía superficial, al eliminar placa o elementos que pudieran interferir en la adhesión ${ }^{(10)}$, reportes de empleo en esmalte de piedra pómez junto con ácido clorhídrico al $18 \%$ muestran un adecuado desempeño, sin embargo, cuando esta es empleada con glicerina la eliminación de partículas se dificulta ${ }^{(11)}$ y por tanto se afecta la capacidad de penetración de los agentes condicionantes y posteriormente adhesivos.

Si bien el estudio no revelo diferencia entre los dos procedimientos de limpieza es necesario considerar que el uso de aire abrasivo requiere de ciertas normas de seguridad para el paciente y el odontólogo deben respetar, sumado al alto costo del equipo y la inaccesibilidad en algunas zonas y la existencia de residuos de polvo en el campo operatorio ${ }^{(12)}$ lo que no ocurre con la piedra pómez y que lleva a pensar en recomendar la piedra pómez como opción, siempre que las partículas de esta sean finas, de baja densidad y peso, lo que permitiría cumplir con su función con una fácil eliminación de sus residuos.

En cuanto a los materiales empleados, los resultados mostraron un comportamiento similar, asociado a su composición (13), acondicionamiento previo ejecutado (14) y cuidados de manipulación, estudios previos revelaron un excelente comportamiento de estos materiales resaltando una mayor filtración marginal a nivel cervical (15)(16), pues resulta innegable el hecho de que al ser ambos de polimerización química, carecen de refuerzo resinoso lo que explicaría el por qué ninguna superficie presentó grado de 0 de micro filtración, lo que se complementa con la acción que el proceso de termociclado desencadena.

Al haber confeccionado las cavidades en el límite amelocementario, el 0 esmalte y dentina fueron tejidos inmiscuidos, a este nivel el esmalte es muy escaso con un espesor de $0,8 \mathrm{~mm}$ lo que nos conduce directamente a la dentina la cual por su compleja estructura lograr en ella la adhesión se considera más difícil que a nivel de esmalte, debido a que la dentina está íntimamente conectada con el tejido pulpar por medio de túbulos dentinarios que se encuentran llenos de líquido el cual se va a desplazar, desde la pulpa a la unión amelodentinaria, es por esto que para lograr una eficaz adhesión, se debe acondicionarla exponiendo sus fibras de colágeno, humedecerla con sustancias hidrofílicas y adherir a ella sustancias hidrófugas , para evitar microfiltración marginal ${ }^{(17-18)}$

Se concluye que hay evidencia de microfiltración en todos los grupos estudiados independientemente del tratamiento de limpieza utilizado y el material de restauración empleado. Así mismo, se evidencio que existe mayor grado de microfiltración en la pared cervical de las preparaciones de todos los grupos.

Sería interesante realizar nuevos estudios evaluando la eliminación del barrillo dentinario y su influencia en la adhesión dentinaria, al estéreo microscópico. 


\section{Contribuciones de autoría}

EMM y AAV Participaron en el diseño del estudio, revisión de la literatura, recolección y análisis de datos, redacción del artículo. AC: Brindó asesoría estadística, redacción y revisión crítica del artículo. Todos aprobaron la versión final del manuscrito.

Fuente de financiamiento: Autofinanciado.

Conflictos de interés: No existe conflicto de intereses en este manuscrito.

\section{REFERENCIAS BIBLIOGRÁFICAS}

1. Barceló F, Sánchez J, Serrano C, Guerrero J. Estudio comparativo de ionómeros de vidrio y reforzados con metal. ADM. 1999; 6(5): 177-181.

2. Litonjua L, Andreana S, Bush P, Cohen R. Tooth wear: Attrition, Erosion, and Abrasion. Quintessence Int.I. 2003 Junio; 34(6):435-46.

3. Rees JS JP. The effect of interfacial failure around a class $\mathrm{V}$ composite retauration analysed by the finite element method. J Oral Rehabil. 2001 December; 27(2):111-116.

4. Hidalgo- Lostaunau R MRM. Ionomeros de vidrio convencionales como base en la técnica restauradora de sándwich cerrado: su optimización mediante la técnica de acondicionamiento ácido simultáneo y selectivo. Acta Odontol Venez. 2009; 47(4): 273-276.

5. Lahod V. Cementos a base de vidrio ionomero. Odontología Sanmarquina. 1999; 1(1): 1-3.

6. Calabria $H$. Lesiones no cariosas del cuello dentario: patología moderna, antigua controversia. Odontoestomatología. 2009 Mayo ; 11(12): 177.

7. Gil. M GG,LD. Terapéutica de las lesiones cervicales. Caracas : Fac. Odontología. UCV; 2013 Febrero.
8. Pereira I, Belkiss F, Silva J, Conceição R, Silami C, Mantovani F, et al. Influence of surface treatment on the performance of silorane-based composite resin in class I restorations: a randomized clinical trial. Clin Oral Invest. 2018 Febrero:1-8.

9. kumar U, Dharmani C, Singh S, Logani A, Shah N. Effect of Air Abrasion Preconditioning on Microleakage in Class V Restorations Under Cyclic Loading: An In-vitro Study. Journal of Clinical and Diagnostic Research. 2014 Mayo; 8(5): 29-32.

10. Fu $B$, Hanning M. Effects of air abrasion and acid etching on the microleakage of preventive Class I resin restorations: an in vitro study. J Esthet Dent.. 1999; 11(3):143-8.

11. Arezoo J, Hamideh A, Mostafa S. Management of Postorthodontic White Spot Lesions and Subsequent Enamel Discoloration with Two Microabrasion Techniques. J Dent (Shiraz). 2015 Mar; 16(1): 56-60.

12. Treviño E. Microabrasion y Operatoria dental. ADM. 2000; 7((3):102-108.

13. Crim C. Marginal leakage of visible light-cured glass ionomer restorative materials. J. Prost. Dent. 1993; 69: 561-563.

14. Jiménez A, Yamamoto A. Valoración de la microfiltración del ionómero de vidrio mejorado (Ketac Molar Easymix (B) con o sin el uso de acondicionador. Rev Odont Mex. 2015; 19(3) :170-173.

15. Kleinman L, Anabalón A, Cortés P, Stanke C. Termociclado en la microfiltración de restauraciones cervicales de resina. Rev. dent. Chile. 1997; 88(3):7-10.

16. Basurto K, Barragán N. Comparación de la

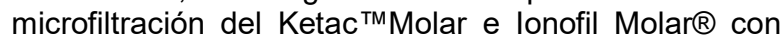
centrix y espátula TRA. CES Odont. 2016; 29 (2): 5-11.

17. Garrofé A, Martucci D, Picca M. Adhesión a tejidos dentarios. Rev. Fac. de Odon. UBA. 2014; 29(67):5-13.

18. Salami D, Alves de Cerqueir M. Effect of prophylactic treatments on the superficial roughness of dental tissues and of two esthetic restorative materials. Pesqui. Odontol. 2003 Mar; 17(1): 63-8. 